\title{
A Linear Complementarity Numerical Approach to the Non-Convex Problem of Structures Environmentally Damaged and Strengthened by Cable-bracings
}

\author{
Dedicated to Academician Blagovest Sendov's 80th anniversary \\ Konstantinos Liolios*, Stefan Radev ${ }^{\dagger}$, Asterios Liolios ${ }^{\ddagger}$, Ivan Georgiev ${ }^{\S}$, Krassimir Georgiev $\rrbracket^{\Uparrow}$ \\ ${ }^{*}$ Department of Environmental Engineering, Democritus University of Thrace, Xanthi, Greece \\ kliolios@env.duth.gr \\ ${ }^{\dagger}$ Institute of Mechanics, Bulgarian Academy of Sciences, Sofia, Bulgaria \\ stradev@imbm.bas.bg \\ $\ddagger$ Department of Civil Engineering, Democritus University of Thrace, Xanthi, Greece \\ aliolios@ civil.duth.gr \\ $\S$ Institute of Mathematics and Informatics, Bulgarian Academy of Sciences, Sofia, Bulgaria \\ john@ parallel.bas.bg \\ IInstitute of Information and Communication Technologies, Bulgarian Academy of Sciences, Sofia, Bulgaria \\ georgiev@parallel.bas.bg
}

Received: 1 August 2012, accepted: 10 December 2012, published: 29 December 2012

\begin{abstract}
A computational treatment is presented for the mathematically rigorous analysis of civil engineering structures, which have been environmentally damaged and subsequently strengthened by cable-elements. The problem is treated as an inequality one, where the governing conditions are equalities as well as inequalities. The cable behavior is considered as nonconvex and nonmonotone one and is described by generalized subdifferential relations including loosening, elastoplastic - fracturing and other effects. Using piece-wise linearization for the cable behavior, a linear complementarity problem, with a reduced number of unknowns, is solved by optimization algorithms. Finally, an example from Civil and Environmental Engineering praxis is presented.
\end{abstract}

Keywords-Civil and Environmental Engineering; NonConvex Analysis; Computational Mechanics; Cable-braced structures; Optimization Algorithms;

\section{INTRODUCTION}

Environmental actions can often cause significant damages to civil engineering structures, see e.g. [1], [2]. Main such defect is the strength degradation, causing a reduction of the load bearing capacity. To handle such defects, sometimes cable-like members are used as a first strengthening and repairing procedure. These cable-like members can undertake tension but buckle and become slack and structurally ineffective when subjected to a sufficiently large compressive force. Thus the governing conditions take an equality as well as an inequality form and the problem becomes nonlinear. So, the problem of structures containing as above cable-like members belongs to the so-called Inequality Problems of Mechanics, as their governing conditions are of both, equality and inequality type [3]-[6] .

A realistic numerical treatment of such problems 
can be obtained by mathematical programming methods (optimization algorithms) [3]-[5]. The early numerical realizations of these approaches were based mainly upon the principle of minimum complementary energy. Thus, an equivalence principle for the analysis of statically undetermined structures with unilateral constraints has been proposed and proven by G. Nitsiotas [3].

Further, for the rigorous mathematical investigation of the problem, convex analysis and the variational or hemivariational inequality concept have been introduced and used, see Panagiotopoulos [6], [7] and [11].

The aim of this paper is to deal with the development of a simple numerical procedure for the rigorous analysis of civil engineering structures containing cablelike members by using a version of the direct stiffness (displacement) method of structural analysis. The present procedure is based on the finite element method and the equivalence principle, proposed by G. Nitsiotas in [3]. Using this principle, the analysis of such structures can be reduced to a Linear Complementarity Problem (LCP), which can be solved by various effective quadratic programming algorithms [5]. A numerical example shows the direct applicability on the computer and the effectiveness of the procedure presented herein.

\section{Method of AnALYsis}

\section{A. Problem formulation}

A frame structure containing $\mathrm{N}$ cable-like members is considered. The structure is discretized according to the natural finite element method [4], [6]. For the cables, pin-jointed bar elements with unilateral behavior are used. Following Panagiotopoulos [6], [7]. the behavior of the cables, including loosening, elastoplastic or/and elastoplastic-softening-fracturing and unloading - reloading effects, can be expressed mathematically by the subdifferential relation:

$$
s_{i}\left(d_{i}\right) \in \hat{\partial} S_{i}\left(d_{i}\right)
$$

Here $s_{i}$ and $d_{i}$ are the (tensile) force and the deformation (elongation), respectively, of the $i$-th cable element, $\hat{\partial}$ is the generalized gradient and $S_{i}$ is the superpotential function [6], [7], [10], [11]. By definition, relation (1) is equivalent to the following hemivariational inequality, expressing the Virtual Work Principle for inequality problems:

$$
S_{i}^{\uparrow}\left(d_{i}, e_{i}-d_{i}\right) \geq s_{i}\left(d_{i}\right) \cdot\left(e_{i}-d_{i}\right)
$$

Here $S_{i}^{\uparrow}$ denotes the subderivative of $S_{i}$, and $e_{i}, d_{i}$ are kinematically admissible (virtual) deformations. For numerical treatments of practical inequality problems, a piece-wise linearization is usually applied to relations (1) and (2), see e.g. [4], [8]. So, the unilateral behavior for the $i$-th cable-element $(i=1, \ldots, N)$ is expressed by the following relations [3], [4]:

$$
\begin{gathered}
e_{i}=F_{0 i} s_{i}+e_{i 0}-v_{i} \\
s_{i} \geq 0, \quad v_{i} \geq 0, \quad s_{i} \cdot v_{i}=0 .
\end{gathered}
$$

Here $e_{i}, F_{0 i}, s_{i}, e_{i 0}$, and $v_{i}$ denote the strain (elongation), natural flexibility constant, stress (tension), initial strain and slackness, respectively. From (3) it is clear that the slackness $v_{i}$ can be considered as an unknown initial strain which constitutes a reversible negative elongation [3]. Further, relations (4) express that either a nonnegative stress-tension or a non- negative slackness exists on any cable.

For the remaining structure (besides the cables), the usual frame finite element models, which exhibit a bilateral behavior, are used.

\section{B. Numerical solution approach}

Now the equivalence principle, proposed by G. Nitsiotas in [3], is applied for the whole structure. According to this principle, the structure under consideration behaves as an equivalent, linearly elastic structure, under the condition that in each cable-element either a nonnegative stress or a fictitious, unknown, non- negative slackness appears-see relations (3). Thus, collecting in $(N \times 1)$ vectors $t$ and $v$ the stress and slackness behavior of all the $N$ cable-elements, corresponding, the following Linear Complementarity Conditions hold:

$$
t \geq 0, \quad v \geq 0, \quad t^{T} v=0 .
$$

Further, following the Stiffness (Displacement) Method of Structural Analysis, we consider the cableelement as solidified rods and we assume that the so-modified structure is a statically stable one with bilateral rod-elements. So, the tension vector $t$ is decomposed as follows [9]:

$$
t=C v+t_{0}
$$

Here $t_{0}$ is the stress vector of the solidified cableelements, now acting as normal bilateral rods, due to external actions and $C$ is the natural influence matrix of $v$ on $t$. For both it is assumed a linearly elastic, bilateral behavior for the stable structure, where. the cables are considered as already solidified bars. So, the natural 
stiffness matrix $C$ is symmetric and in general positive semi-definite.

Thus, if $t_{0}$ is known, then vectors $t$ and $v$ can be determined by solving the Linear Complementarity Problem (LCP) formed by relations (5) and (6). For the solution of this problem, various effective algorithms are available [3]-[10]. Most of these algorithms reduce the above linear complementarity problem to a quadratic programming one of the form:

$$
\operatorname{Min}\left\{\frac{1}{2} v^{T} C v+v^{T} t_{0}: v \geq 0\right\}
$$

After the previous preparation we can now formulate the following numerical procedure for the analysis of structures containing cable like members:

a) Considering the cables as having been solidified (normal bilateral bars), the vector $t_{0}$ due to external actions is determined by the Finite Element Method.

b) Under the same assumption and by the same method as in a), the influence matrix $C$ is determined. In this matrix, $C_{i j}$ is the stress (axial force) in the solidified cable-element $i$ caused by a unit-shortening $v_{j}=1$ imposed in the solidified cable-member $j,(i, j=$ $1, \ldots, N)$.

c) The Linear Complementarity Problem of relations (5) and (6) is solved to provide the sought vector $v$. So it is computed which cable-elements are activated (under tension) and which are not (under non-zero slackness).

d) The final stress state of the structure is determined by taking into account the external actions and the computed forces $t$ of the active cable-elements.

Thus, the whole procedure requires the linear elastic analysis of the modified (with solidified cable-elements) structure $(N+2)$ times, where $N$ is the number of the cables, and the solution of a quadratic programming problem or a LCP. Alternatively, after having computed $t$, the structure is analyzed due to external actions by omitting the slack cables for which the step c) has given zero tension values.

\section{NUMERICAL EXAMPLE}

The reinforced concrete plane frame structure of Fig. 1 had been initially analyzed, designed and constructed to bear the shown loads, without the shown cableelements. The concrete class is $\mathrm{C} 40 / 50$ and the elasticity modulus $E_{b}=3.5 \times 10^{7} \mathrm{kN} / \mathrm{m}^{2}$. The shown dimensions width/height of rectangular sections are in centimeters $[\mathrm{cm}]$.
The analysis of the above frame without cableelements is obtained by using any available finite element method code, e.g. SAP2000 code [13].

Due to environmental actions [1], [2], corrosion and cracking had been taking place. This had caused a reduction for the section inertia moments, which, according to [14], is estimated to be $10 \%$ for the columns and $50 \%$ for the beams. So it was necessary for the system to be strengthened. As a first repairing and strengthening procedure, ten $(N=10)$ cable members with crosssectional area $F_{r}=8 \mathrm{~cm}^{2}$ have been added as shown in Fig. 1. The steel class is $\mathrm{S} 1400 / 1600$ with an elasticity modulus $E_{s}=210 G P a$. These cables are placed as counter diagonals. As it is not known in advance which of them are activated or not by the given loads, the purpose here is to compute what happens.

The application of the presented numerical procedure gives first the values of the slackness of the not activated cable-elements:

$$
\begin{aligned}
& v_{1}=0.848 \times 10^{-3} \mathrm{~m}, v_{3}=10.321 \times 10^{-3} \mathrm{~m}, \\
& v_{5}=1.082 \times 10^{-3} \mathrm{~m}, v_{8}=9.564 \times 10^{-3} \mathrm{~m}, \\
& v_{10}=1.652 \times 10^{-3} \mathrm{~m} .
\end{aligned}
$$

Further, the elements of vector $t$, where

$$
t=\left[S_{1}, S_{2}, \ldots, S_{10}\right] T,
$$

are computed to have the following values (in $\mathrm{kN}$ ) for the non-active cables:

$$
S_{1}=S_{3}=S_{5}=S_{8}=S_{10}=0.0,
$$

whereas for the active cables it holds:

$$
S_{2}=10.17 k N, S_{4}=346.04 k N, S_{6}=18.84 k N,
$$$$
S_{7}=342.08 k N, S_{9}=25.81 k N \text {. }
$$

Thus, cables 2,4,6,7 and 9 are the only ones active, having zero slackness. The other cables $1,3,5,8$ and 10 cannot contribute to the system resistance under the given loads in Fig. 1.

Using the previous results, the final stress state is computed. In Fig. 2 is shown indicatively the final bending moments diagram for the strengthened frame containing the active cable-elements only. Comparing the diagram in Fig. 2 with the corresponding one for the initial frame without cables, the efficiency of the strengthening can be checked.

\section{CONCLUSION}

The inequality problem of the cable-braced civil engineering structures can be treated numerically by the herein presented approach. This approach takes into account the unilateral behavior of cable elements, uses the equivalence principle of G. Nitsiotas [3] and so leads to a linear complementarity problem, with a reduced number of problem unknowns. Thus, the numerical realization of 


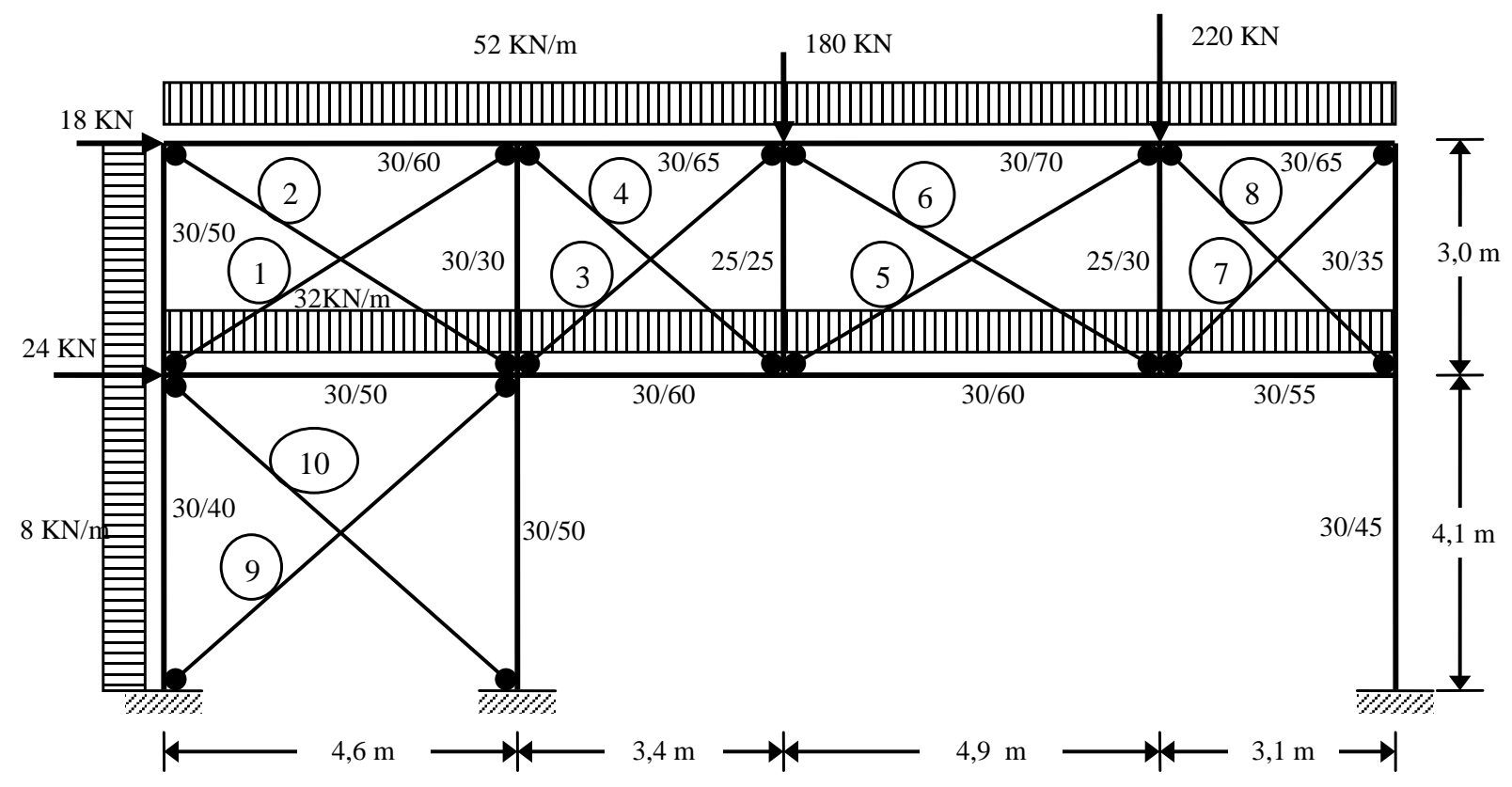

Fig. 1. The cable-braced structural system of the numerical example.

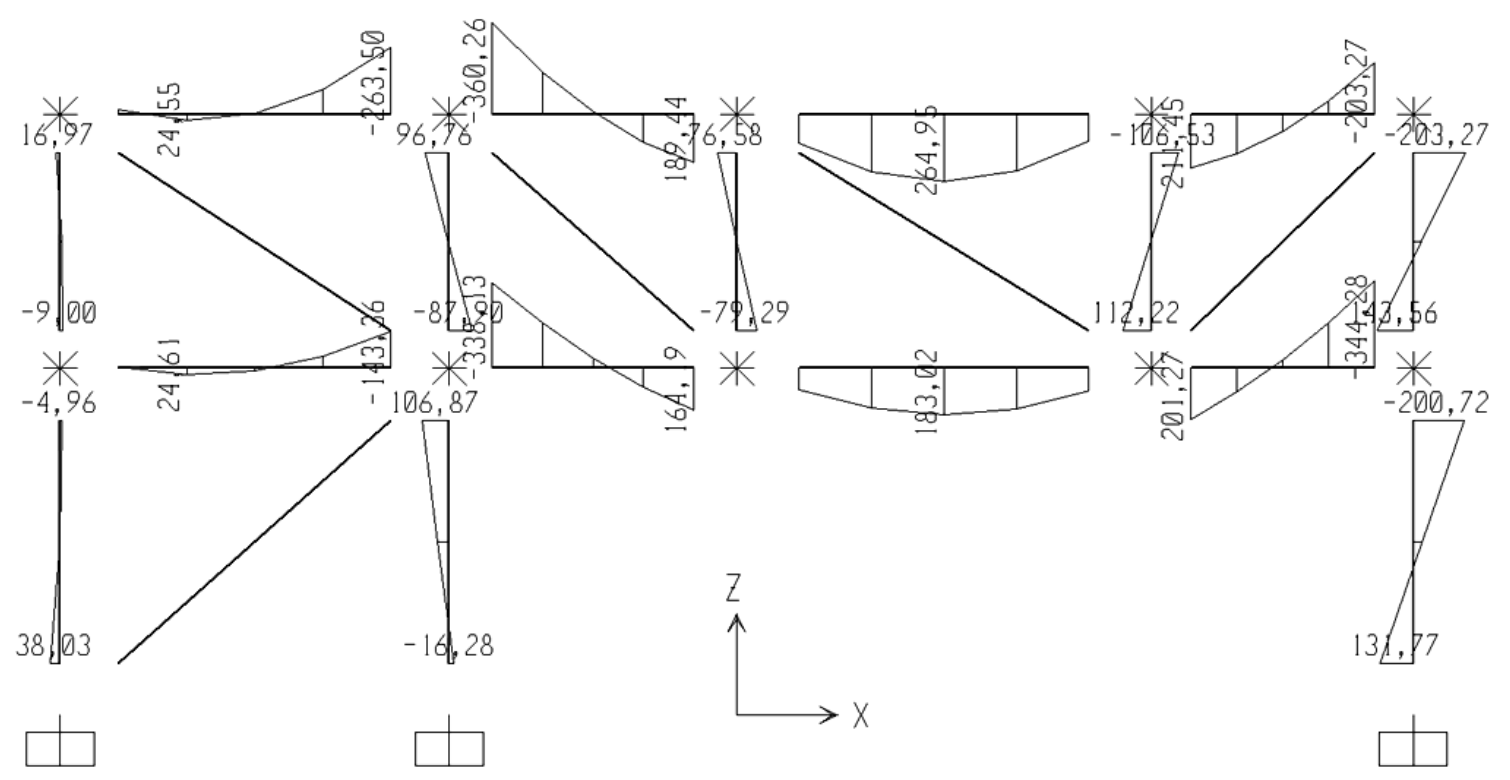

Fig. 2. Bending moments diagram (in $k N m$ ) for the frame with the 5 active cable-elements No 2,4,6,7 and 9. 
the proposed approach is obtained by available computer codes of the finite element method and of mathematical programming algorithms. Moreover, as it has been verified in an example, the herein developed approach can treat in a realistic way the general problem of cablebraced structures in civil and environmental engineering praxis. An extension of the presented approach for the case of the earthquake response of such structures has been recently presented [12].

\section{ACKNOWLEDGMENT}

This work was partially supported by the Bulgarian Science Fund under the project DMU 03-62.

\section{REFERENCES}

[1] H. S. Peavy, D. R. Rowe, and G. Tchobanoglous, Environmental Engineering, McGraw-Hill, New York, 1985.

[2] A. Moncmanova, Environmental Deterioration of Materials, WIT Press, Southampton, 2007. http://dx.doi.org/10.2495/978-1-84564-032-3

[3] G. Nitsiotas, Die Berechnung statisch unbestimmter Tragwerke mit einseitigen Bindungen, Ingenieur-Archiv, vol. 41, pp. 46-60, 1971.

http://dx.doi.org/10.1007/BF00536162

[4] G. Maier, A Quadratic Programming Approach for Certain Classes of Non-linear Structural Problems, Meccanica , vol. 3, pp. 121-130, 1968. http://dx.doi.org/10.1007/BF02129011

[5] R. W. Cottle, Fundamentals of Quadratic Programming and Linear Complementarity, in M.Z. Cohn, D.E. Grierson and G. Maier (Eds.), "Engineering Plasticity by Mathematical Programming", Pergamon Press, Oxford, 1979.
[6] P. D. Panagiotopoulos, Inequality Problems in Mechanics and Applications. Convex and Nonconvex Energy Functions, Birkhuser Verlag, Basel, Boston, 1985. http://dx.doi.org/10.1007/978-1-4612-5152-1

[7] P. D. Panagiotopoulos, Hemivariational Inequalities. Applications in Mechanics and Engineering, Springer Verlag, Berlin, 1993. http://dx.doi.org/10.1007/978-3-642-51677-1

[8] A. A. Liolios, A Linear Complementarity Approach for the Nonconvex Seismic Frictional Interaction between Adjacent Structures under Instabilizing Effects. Journal of Global Optimization, vol. 17 , no. $1-4$, pp. 259-266, 2000. http://dx.doi.org/10.1023/A:1026789817828

[9] Ang. Liolios, Numerical Simulation of Structures with Unilateral Constraints and Solution by the Finite Element Method, Graduate Degree Thesis, Department of Civil Engineering, Democritus University of Thrace, Xanthi, Greece, 2007.

[10] E. S. Mistakidis and G. E. Stavroulakis, Nonconvex optimization in mechanics. Smooth and nonsmooth algorithmes, heuristic and engineering applications, Kluwer, London, 1998.

[11] R. T. Rockafellar, Convex Analysis, Princeton Univ. Press, Princeton, 1970.

[12] Ang. Liolios, K. Chalioris, Ast. Liolios, St. Radev, and Kon. Liolios, A Computational Approach for the Earthquake Response of Cable-braced Reinforced Concrete Structures under Environmental Actions. In: Lirkov, I., Margenov, S. and Wasniewski, J. (Eds): "Large-Scale Scientific Computing", LNCS 7116, pp. 590-597, Springer-Verlag, Berlin Heidelberg, 2012. http://dx.doi.org/10.1007/978-3-642-29843-1

[13] Computers and Structures, Inc. SAP2000, Integrated Finite Element Analysis and Design of Structures, Berkeley, 2005.

[14] T. Pauley and M. J. N. Priestley, Seismic design of reinforced concrete and masonry buildings, Wiley, New York, 1992. http://dx.doi.org/10.1002/9780470172841 